\title{
Lower Urinary Tract Mesh Perforations following the Insertion of Mid-urethral Tapes or Transvaginal Mesh: A Tertiary Unit's Experience
}

\author{
Dalia Saidan $^{1 *}$, Veenu Tyagi ${ }^{1}$, Paraskeve (Voula) Granitsiotis ${ }^{2}$ and Karen Guerrero ${ }^{1}$ \\ ${ }^{1}$ Queen Elizabeth University Hospital, Glasgow, United Kingdom \\ ${ }^{2}$ Western Infirmary, Edinburgh, United Kingdom
}

*Corresponding author: Dalia Saidan, Queen Elizabeth University Hospital, Glasgow, United Kingdom

\begin{abstract}
Mesh perforation of the urinary-tract is a rare complication of midurethral tapes for stress urinary incontinence (SUI), and transvaginal mesh for pelvic organ prolapse (POP). We review the outcomes of women presenting to our tertiary service.

This retrospective review identifies patients presenting to our unit between January 2011 - June 2017.

We identified 40 women. Twenty-three had perforation from SUI tapes, 10 following POP mesh, and 7 had both. All underwent surgical management. Seven reported being cured, 24 reported improvement, 6 reported no change and 2 reported worsening symptoms.

Seventeen developed worsening post-operative SUI. Ten opted for surgical management. Four had autologous fascial sling (AFS), 4 had bladder neck injections and 2 had colposuspension. Two patients reported recurrence in POP. One was treated successfully with physiotherapy and the other with a sacrocolpopexy.

Treatment of mesh perforation is a complex process. As part of a specialised MDT, complications can be minimised.
\end{abstract}

\section{Keywords}

Mesh, Perforation, Post-operative outcomes, Excision

\section{Introduction}

Synthetic mesh has been used in to treat Pelvic Organ Prolapse (POP) and Stress Urinary Incontinence (SUI) for over 20 years. Use of synthetic mid urethral tapes were hailed as revolutionary when they were first widely used in the 90s [1], leading to them becoming the most commonly performed anti-incontinence operation on women worldwide [2]. Advantages include shorter operating times and arguably good success rates [3].

However, over recent years there has been growing controversy around their use [4]. As public concerns grew in Scotland, the Scottish Government called for a suspension in 2014 and commissioned a review of the use of mesh grafts, the final report of which was published in March 2017 [5]. Since July 2018 in England, the Government has advised of a period of high vigilance restriction, and as such, vaginal mesh surgery should not be performed for the majority of patients during this period [6]. This echoes similar restrictions in other countries; in Australia, vaginal mesh used to treat pelvic organ prolapse has been banned [7].

Mesh implant complications are well recognised, with a formal classification system, published jointly by the International Urogynecological Association (IUGA) and the International Continence Society (ICS) January 2011 [8].

For the purpose of this paper, we have classified perforation according to the IUGA mesh complication system, 'An abnormal opening into a hollow organ or viscus'. [8] In this paper, this relates to patients presenting to our tertiary service at a later date, rather than intra-operative injury identified at the time of surgery.

Perforation of the urinary-tract is a rare but recognised complication of mid-urethral tapes and vaginal mesh grafts $[2,9]$. These can involve any part of the low-

Citation: Saidan D, Tyagi V, Granitsiotis P, Guerrero K (2019) Lower Urinary Tract Mesh Perforations following the Insertion of Mid-urethral Tapes or Transvaginal Mesh: A Tertiary Unit's Experience. Int Arch Urol Complic 5:059. doi.org/10.23937/2469-5742/1510059

Accepted: May 30, 2019: Published: June 01, 2019

Copyright: (C) 2019 Saidan D, et al. This is an open-access article distributed under the terms of the Creative Commons Attribution License, which permits unrestricted use, distribution, and reproduction in any medium, provided the original author and source are credited. 
er urinary tract, namely bladder \& urethra, but ureteric injuries can also occur [8]. The mesh revision surgery and urinary tract reconstruction involved in dealing with these complications is often complex [9] and as a consequence is often centralised in larger centres. The British Society Urogynaecology (BSUG) and British Association of Urological surgeons (BAUS) now list accredited centres in the UK who manage patients with mesh implant complications.

As a regional tertiary centre, our unit accepts referrals for mesh implant complications. Its' Multidisciplinary mesh complication service, being formally established since late 2011, is listed as a mesh complication centre by both British Society of Urogynaecologists (BSUG) and British Association of Urological Surgeons (BAUS).

Our service consists of a team of Urogynaecologists, Urologists, Coloproctologists, Physiotherapists, Pain Physicians and specialist nurses working within a wider multidisciplinary team (MDT) structure of a large teaching hospital.

Services available to mesh patients include conservative management of complications, including pain management services, pelvic floor physiotherapy, as well as surgical management. Surgical procedures offered for mesh complications include complete and partial mesh excision. This can be done abdominally, through open or laparoscopic procedures, or vaginally. As well as laparoscopic excision, other minimal access procedures offered through our MDT service include cystoscopic mesh excision, including laser.

\section{Methods}

This is a retrospective review of patients presenting to our specialised service. Subjects were identified from the unit's audit database between January 2011 - June 2017. Electronic records and case notes were used to collect data.

We collected data surrounding presentation, type of implant, nature of the injury and management. We also looked at surgical outcomes including complications.

Significant peri-operative complications were defined as: blood transfusion, prolonged stay or readmission to hospital for procedure related event, sepsis requiring intravenous antibiotic therapy and post-operative urinary incontinence, including persistent post-operative fistula.

Due to the varied complex nature of the presenting symptoms, for the purpose of this paper we defined patient reported "success" into 4 categories:

1. "Cured": subjects reported resolution of presenting symptoms, required no further treatment and had no new onset symptoms (including pain).

2. "Improved": subjects reported significant improve- ment in their presenting symptoms but had ongoing symptoms requiring further management.

3. "No change": subjects reported no significant change to their presenting symptoms.

4. "Worse": subjects with worsening or new onset symptoms following surgery.

\section{Results}

There was a total of 45 cases of patients referred with urinary tract mesh perforation over this time period. This corresponds to $14 \%$ of all referrals between January 2011 and June 2017 to the service.

We were able to obtain case notes for 40 subjects. All underwent mesh excision.

Subjects' ages ranged from 40 years to 89 years, with a mean age of 59 years and a modal age of 54 years at the time of the referral. The subjects with mesh perforation from mid-urethral tapes were the younger age group with a mean age of 54 years, versus a mean age of 64 years for patients presenting with perforation from transvaginal mesh for the treatment of pelvic organ prolapse.

All subjects had complications classified as IUGA category (8) 4B.

Patients presented with a variety of symptoms, with the majority presenting with multiple symptoms. Twelve (30\%) presented with pain. Pain was usually located in the groin, abdomen or vagina with 5 (42\%) describing pain in more than one location. $16(40 \%)$ subjects presented with overactive bladder symptoms, namely frequency, urgency and urge incontinence.

Six $(15 \%)$ subjects presented with urinary incontinence that was not attributed to overactive bladder; one had visible stress incontinence and 5 (13\%) had symptoms of continuous dribbling, which were confirmed to be fistulae.

Three (8\%) subjects complained of vaginal discharge and another $3(8 \%)$ had recurrent Urinary Tract Infections. Two (5\%) women had dyspareunia and 2 (5\%) presented with frank haematuria. One (3\%) felt the mesh vaginally and another patient complained of chronic fatigue symptoms she attributed to the mesh.

Eighteen (45\%) women had developed symptoms within the first year following implant insertion, however 12 women (30\%) presented over 5 years after graft insertion (Table 1).

The majority of referrals (Table 2) were due to perforations following mid-urethral tapes (58\%) with transobturator tapes being the largest implant type in our series (40\%).

The commonest site of injury was the urethra for mid urethral tapes although bladder injuries were also seen. 
Table 1: Time from implant insertion to onset of symptoms.

\begin{tabular}{|l|l|l|}
\hline $\begin{array}{l}\text { Time from Implant insertion to symptom } \\
\text { development }\end{array}$ & IUGA Classification & Number of patients (\%) \\
\hline$<48$ hours & T1 & 0 \\
\hline$<2$ months & T2 & $8(20 \%)$ \\
\hline 2-12 months & T3 & $10(25 \%)$ \\
\hline 1-3 years & T4 & $5(12.5 \%)$ \\
\hline 3-5 years & T4 & $5(12.5 \%)$ \\
\hline 5+ years & T4 & $12(30 \%)$ \\
\hline
\end{tabular}

Table 2: Type of implant and site of Urinary tract injury.

\begin{tabular}{|l|l|l|l|l|}
\hline Type of Implant & Total & Site of Injury & \multicolumn{2}{l|}{} \\
\cline { 3 - 5 } & & Bladder & Urethra & 0 \\
\hline Transobturator tape & $16(40 \%)$ & 4 & 12 & 0 \\
\hline Retropubic tape & $7(17.5 \%)$ & 3 & 4 & 0 \\
\hline POP vaginal mesh & $10(25 \%)$ & 10 & 0 & 1 \\
\hline Both & $7(17.5 \%)$ & 5 & 1 & 1 \\
\hline
\end{tabular}

For POP vaginal mesh implants the main site of injury was the bladder, with only 1 ureteric injury (Table 2).

\section{Urinary tract mesh perforations following midure- thral tapes}

We identified 23 women with urinary tract perforation following the insertion of midurethral tape (Table 2 ). Sixteen $(70 \%)$ had urethral perforation and $7(30 \%)$ had bladder perforation.

Three out of sixteen women (19\%) with urethral perforation had urethro-vaginal fistulas.

All subjects with urethral perforations underwent surgical management. Fifteen out of sixteen (94\%) had the mesh excised vaginally with urethral reconstruction. One woman opted for cystoscopic management where the mesh perforating the urethra was cut.

A Martius fat graft was used in 7 cases, which included all three cases with a fistula. All grafts were successful with no resultant fistula or defects.

Four out of seven $(57 \%)$ subjects with a bladder perforation from a mid-urethral tape had the mesh excised vaginally. These were the subjects with TOT perforations where the tape was perforating the bladder base and was therefore best accessible via the vaginal route. In the retropubic tape perforations (3 subjects), one $(14 \%)$ had cystoscopic removal of the mesh perforating into the bladder dome. The remaining two (29\%) required abdominal cystotomy and vaginal excision of mesh, to excise all of the implanted mesh.

There were no significant peri or post-operative complications in women undergoing excision for midurethral tape perforations in the urinary tract. No subject has presented with further mesh perforations or exposures.

\section{Urinary tract perforations following vaginal mesh implants used to treat pelvic organ prolapse}

We identified 10 women with perforation following transvaginal mesh insertion to treat pelvic organ prolapse. All had bladder perforations following insertion of anterior compartment mesh. This included 2 subjects presenting with vesico-vaginal fistula. Surgical approach was dictated by site of perforation into the bladder as well as patients' wish for extent of implant removal.

Three (30\%) subjects had abdominal cystotomy for resection of the perforated mesh from the bladder, without full implant removal, as they declined extensive mesh removal surgery.

Three (30\%) subjects had complete vaginal excision of the mesh with vaginal cystotomy and repair. The two fistula patients are included in this group.

Two (20\%) had partial excision of the mesh vaginally, to repair defect. Both of these women, however, had persisting mesh fibres in the bladder, which were subsequently removed by cystoscopic laser.

Two subjects (20\%) had a combined vagino-abdominal approach to resect all the mesh.

There were no subjects in our case series with urethral injuries following transvaginal mesh insertion alone.

There were no other significant peri or post-operative complications in patients having mesh excision following vaginal mesh implants used to treat pelvic organ prolapse.

\section{SUI \& POP grafts}

Seven (18\%) subjects had both midurethral tapes and vaginal mesh inserted. All SUI implants in this group were transobturator tapes, combined with anterior 
transvaginal mesh grafts.

Of these, 5 (71\%) had a bladder perforation and 1 (14\%) had a urethral perforation. All the bladder perforations were found to be from transvaginal mesh but the urethral perforation was due to the concomitant tape.

Three (60\%) of the subjects with bladder perforation had complete excision of both vaginal mesh implants. One of the subjects, however, who was thought to have complete mesh excision vaginally, presented with persistent $O A B$ symptoms at follow up. Subsequent cystoscopy showed some mesh fibres in the bladder which were excised cystoscopically with symptom resolution.

One subject had a partial excision by an abdominal cystotomy. She opted to have only the perforating portion of the mesh excised and recovered well.

One subject had partial excision vaginally of the perforating mesh. This healed well, however, represented with a further mesh perforation in the bladder, which required endoscopic laser treatment.

The subject with the urethral injury had cystoscopic excision.

One (14\%) subject presented with a fistula one month post-operatively having sustained a ureteric injury from an anterior mesh, not the mid-urethral tape implant concomitantly inserted. The subject had a vaginal excision of the mesh and abdominal surgery for reimplantation of the ureter.

\section{Follow-up}

All of our patients received post-operative physiotherapy to optimise their recovery and to minimise the possibility of recurrent pelvic organ prolapse or stress urinary incontinence.

We followed patients up initially at 3- months post-operatively. Follow up appointments after this depended on individual patient needs.

There was no follow up information for one subject who underwent excision secondary to perforation. She had a bladder perforation following retropubic tape. She had a complete combined vagino-abdomino excision. She did not attend her follow up appointment, and no further information is available on this subject.

\section{Patient reported outcomes}

Following excision of mesh grafts for perforation of the urinary tract, the majority of subjects saw benefit (Table 3): Overall, 7 (18\%) subjects reported being cured and 24 (60\%) reported that they had improved. Six (15\%) subjects, however reported no change in symptoms and 2 (5\%) subjects reported worsening symptoms.

From our data set, there does not appear to be a difference in the outcome depending on the type of mesh inserted. Our numbers are too small for a subgroup analysis for further evaluation.

Looking specifically at pain (Table 4), twelve out of forty $(30 \%)$ subjects reported pain at presentation. Pain was the reason that two of the subjects (5\%) reported worsening symptoms post excision: One developed worsening chronic pain following complete mesh excision of her TOT mesh. The other subject developed new onset pain and chronic fatigue syndrome following excision of her anterior mesh.

In the 30 subjects who had tape related surgery (all tape patients and patients with combined POP and SUI grafts), 17 (57\%) women developed worsening postoperative SUI.

Seven out of seventeen subjects (41\%) after physiotherapy opted for no further management. The remain-

Table 3: Patient reported outcomes.

\begin{tabular}{|l|l|l|l|l|l|l|}
\hline \multirow{2}{*}{ Type of mesh } & \multicolumn{3}{l|}{ Outcome following excision } & \multicolumn{2}{l|}{} \\
\cline { 2 - 7 } & Total no of patients & Cured & Improved & No Change & Worse & No FU data \\
\hline Transobturator Tape & 16 & $3(19 \%)$ & $10(63 \%)$ & $2(12 \%)$ & $1(6 \%)$ & 0 \\
\hline Retropubic Tape & 7 & $1(17 \%)$ & $5(71 \%)$ & 0 & 0 & 1 \\
\hline POP vaginal mesh & 10 & $2(20 \%)$ & $5(50 \%)$ & $2(20 \%)$ & $1(10 \%)$ & 0 \\
\hline Both & 7 & $1(14 \%)$ & $4(57 \%)$ & $2(29 \%)$ & 0 & 0 \\
\hline Total & 40 & $7(18 \%)$ & $24(60 \%)$ & $6(15 \%)$ & $2(5 \%)$ & $1(2 \%)$ \\
\hline
\end{tabular}

Table 4: Outcomes post excision surgery in patients with pain symptoms.

\begin{tabular}{|l|l|l|l|l|l|l|}
\hline Type of mesh & \multicolumn{3}{|l|}{ Outcome following excision } & \multicolumn{2}{l|}{} \\
\cline { 2 - 7 } & Total number & Cured & Improved & No Change & Worse & No FU data \\
\hline Transobturator Tape & $7(58 \%)$ & 0 & $4(57 \%)$ & $1(14 \%)$ & $1(14 \%)$ & $1(14 \%)$ \\
\hline Retropubic Tape & $3(25 \%)$ & 0 & $2(67 \%)$ & 0 & 0 & $1(33 \%)$ \\
\hline POP vaginal mesh & $1(8.5 \%)$ & 0 & 0 & 0 & $1(100 \%)$ & 0 \\
\hline Both & $1(8.5 \%)$ & 0 & 0 & $1(100 \%)$ & 0 & 0 \\
\hline
\end{tabular}


ing ten subjects (59\%) opted to have further surgical management following failure to improve sufficiently with pelvic floor muscle training: four (40\%) underwent a successful AFS and 4 (40\%) had bladder neck injections (BULKAMID) and did not require further management. Two subjects (20\%) opted for a colposuspension; This was not successful in 1 woman (50\%) who then went on and had an AFS which did work for her.

Only two out of seventeen (12\%) subjects who had excisions of transvaginal mesh inserted to treat vaginal prolapse reported a recurrence of prolapse symptoms at follow-up. One was treated successfully with physiotherapy and the other subject (50\%) opted for further surgical management; she underwent an abdominal sacrocolpopexy for recurrent apical prolapse.

\section{Discussion}

Management of urinary tract mesh perforations is a complex process, often because not all perforations are the same and patients often opt for different management depending on symptoms, expectations and belief. Mesh revision surgery can require bladder repair or urethral reconstruction, or ureteric implantation depending on the site and extent of the injury.

We have shown that removal of implants and urinary tract injury repair can be successful with relatively low intra \& post-operative morbidity. However, success depending on symptom resolution for patients is different.

Mesh perforation to the urinary tract is a rare complication.

As we offer a national service, we have relatively larger numbers that we can report on. Furthermore, the numbers of publications reporting urinary tract perforation following transvaginal mesh for POP are limited. Although still moderate in size, we have reported on a larger case series looking at mesh perforations following mid-urethral tapes and transvaginal mesh grafts than have previously been published [10]. We have a larger case series of both SUI and POP mesh but also POP combined with SUI mesh. This is a strength of our paper.

There appear to be two peak periods for patients presenting with perforation. One within the first 12 months (45\%) of mesh placement and one after 5 or more years (30\%). The nature of the complication arising at this late stage may be attributable to mesh 'eroding' through the tissue planes and perforating over time. It is likely that the presentations within 12 months of placement were potentially missed intraoperative injuries, and therefore different in aetiology than the ones presenting years after insertion in women who have been asymptomatic until then.

There was no significant difference seen in the post excision outcomes of subjects, depending on the type of mesh that they had; the majority of subjects reported being "cured or "improved", in both mesh groups.

A weakness of this study is the lack of validated outcome measures and the reliance solely on reported subjective outcomes

Interestingly, in the patients with recurrent stress urinary incontinence who did proceed with further surgery ( $59 \%$ of those with recurrent symptoms), all AFS procedures were successful.

This was not the case for one of the patients who had a Burch type colposuspension (50\%), who required a sling procedure following failed colposuspension. The numbers are far too small to show a significance or draw definitive conclusions. However, it appears that AFS is a better option for patients requiring treatment following midurethral mesh excision. Shah et al. reported a $71 \%$ continence rate and Kowalick et al. reported 92\% continence rate for AFS post mesh removal and reconstruction $[9,11]$.

$68 \%$ of Kowalick's, et al's case series had a concomitant SUI procedure post mesh excision, suggesting they preferred a one-stage approach.

Our case series has shown that not all women require further SUI surgery, and we would therefore advocate a two-stage approach. This may give better functional results, but most importantly, avoid additional procedures that may not improve outcome on women.

There are limited data regarding recurrence of original prolapse symptoms and need for further surgical intervention following mesh excision.

Uptake of cystoscopic management is low in our unit. We therefore accept we may not have a representative cohort and cannot comment on cystoscopic treatment from this case series. From our experience, this treatment approach seemed to be effective in managing residual mesh fibres seen cystoscopically following removal, rather than for managing extensive perforations.

Our learning curve is also demonstrated in our results. As surgical experience for implant removal grew as a whole, and our early data showed increased recurrent perforations with a more conservative approach, consideration is now given to remove all implant. This view is shared by Shah et al. [11]. The options of both are discussed in detail as we counsel our patients on surgical management. There have been no residual fibres in our later cases.

Perforations due to POP mesh are arguably more complex than for SUI, as are patients with both grafts. This is reflected in our paper by the procedures performed and our outcomes, with laser revision required in our earlier cases.

There is very little laparoscopic management of mesh perforation in this cohort. We do offer laparoscopic approach to excise retropubic tapes and abdominal pelvic 
organ prolapse meshes. We acknowledge some of the abdominal cystotomies performed early in our case series would now be offered a laparoscopic approach.

\section{Conclusion}

Management of perforations from vaginal mesh grafts for both SUI and POP is a complex process. As part of a specialised MDT, complications can be minimised, and outcomes improved.

The majority of patients with mesh perforation had excision of the mesh and reported favourable outcomes. Worsening pain following surgery is a possibility.

Recurrent SUI is common following midurethral mesh excision. However, not all symptomatic women require further SUI surgery. For those who do, we prefer placement of AFS.

\section{Ethical Approval}

No ethical approval was required as this is a retrospective case note review of standard medical care undertaken in our unit.

\section{References}

1. Oliphant SS, Wang L, Bunker CH, Lowder JL (2009) Trends in stress urinary incontinence inpatient procedures in the United States, 1979-2004'. Am J Obstet Gynecol 200: e1e6.

2. Blaivas JG, Purohit RS, Benedon MS, Mekel G, Stern M, et al. (2015) Safety considerations for synthetic sling surgery. Nat Rev Urol 12: 481-509.

3. European Commission, Scientific Committee on Emerging and Newly Identified Health Risks (SCENIHR) (2015) Preliminary opinion on the safety of surgical meshes used in urogynaecological surgery (consultation).

4. United States Food and Drug Administration (2011) Urogynecologic surgical mesh: Update on the safety and effectiveness of transvaginal placement for pelvic organ prolapse.

5. Scottish Government (2017) Scottish Independent Review of the use, safety and efficacy of transvaginal mesh implants in the treatment of stress urinary incontinence and pelvic organ prolapse in women: Final Report.

6. RCOG (2018) Mesh safety alert.

7. Therapeutic Goods Administration (2018) TGA actions after review into urogynaecological surgical mesh implants.

8. Haylen BT, Freeman RM, Swift SE, Cosson M, Davila $\mathrm{GW}$, et al. (2010) An international urogynecological association (IUGA)/International continence society (ICS) joint terminology and classification of the complications related directly to the insertion of prostheses (meshes, impplants, tapes) and grafts in female pelvic floor dysfunction. Neurourol Urodyn 30: 2-12.

9. Kowalik CG, Cohn JA, Kakos A, Lang P, Reynolds WS, et al. (2018) Road to recovery after transvaginal surgery for urethral mesh perforation: Evaluation of outcomes and subsequent procedures. Int Urogynecol J 29: 887-892.

10. H Bekarma, $P$ Granitsiotis (2017) The one-year experience of tape and mesh removal at a urological tertiary referral centre. Journal of Clinical Urology 10: 336-339.

11. Shah K, Nikolavsky D, Gilsdorf D, Flynn BJ (2017) Surgical management of lower urinary mesh perforation after mid-urethral polypropylene mesh sling: mesh excision, urinary tract reconstruction and con-comitant pubovaginal sling with autologous rectus fascia. Int Urogynecol $\mathrm{J} 24$ : 2111-2117. 